\title{
Analytic Properties of the Teukolsky Equation *
}

\author{
J. B. Hartle and D. C. Wilkins \\ Department of Physics, University of California, \\ Santa Barbara, California, USA
}

Received January 2, 1974

\begin{abstract}
The analytic properties of the solutions to the Teukolsky equation in the complex frequency plane are investigated. The scattering coefficient $Z_{1 \mathrm{n}}$ is found to be an analytic function of the frequency except at singularities and at certain branch points in both the upper and lower frequency plane. The implications for the proof of the stability of the Kerr geometry given by Press and Teukolsky are discussed.
\end{abstract}

\section{Introduction}

In 1972 Teukolsky [1] derived a separable wave equation whose solutions describe the dynamical gravitational, electromagnetic, scalar, and neutrino field perturbations of a Kerr rotating black hole. Press and Teukolsky [2] have applied this equation to an investigation of the stability of the Kerr geometry under small gravitational perturbations. Their analysis depends on an understanding of the analytic properties of the solutions to the Teukolsky equation in the complex frequency plane. In this paper we will derive the relevant analytic properties.

The Teukolsky equation is a linear equation for a perturbation quantity $\psi$ of spin-weight $s$. In the case of gravitational perturbations, for example, $\psi$ would be the component $\psi_{0}$ or $\psi_{4}$ of the Weyl tensor in Newman-Penrose notation. In Boyer-Lindquist coordinates, the equation can be separated by breaking $\psi$ into components which vary harmonically in time $[\exp (-i \omega t)]$ and by expanding it in terms of spheroidal harmonics ${ }_{s} \mathscr{S}_{l}^{m}(\theta, \varphi)$ of spin-weight $s$. The resulting equation for the radial part $R$ of $\psi$ describing the perturbations of a Kerr geometry of mass $M$ and specific angular momentum $a$ takes the form

$$
\frac{1}{\Delta^{s}} \frac{d}{d r}\left(\Delta^{s+1} \frac{d R}{d r}\right)+\left[\frac{K^{2}-2 i s(r-M) K}{\Delta}+4 i s \omega r-\lambda\right] R=0
$$

where $K=\left(r^{2}+a^{2}\right) \omega-a m, \quad \Delta=r^{2}-2 M r+a^{2}=\left(r-r_{+}\right)\left(r-r_{-}\right)$, and $\lambda=a^{2} \omega^{2}-2 a m \omega+A(a \omega)$. Here, $A(a \omega)$ is the separation constant of the

\footnotetext{
* Supported in part by the National Science Foundation.
} 
angular equation for the functions ${ }_{s} S_{l}^{m}(\theta)$ defined by ${ }_{s} \mathscr{S}_{l}^{m}(\theta, \varphi)$ $=e^{-i m \varphi} S_{l}^{m}(\theta)$. Written out, this equation reads

$$
\begin{gathered}
\frac{1}{\sin \theta} \frac{d}{d \theta}\left(\sin \theta \frac{d S}{d \theta}\right)+\left[a^{2} \omega^{2} \cos ^{2} \theta-\frac{m^{2}}{\sin ^{2} \theta}-2 a \omega s \cos \theta\right. \\
\left.-\frac{2 m s \cos \theta}{\sin ^{2} \theta}-s^{2} \cot ^{2} \theta+s+A\right] S=0 .
\end{gathered}
$$

The quantity $A$ is determined by the requirements of regularity for $S$ at $\theta=0$ and $\theta=\pi$. There are a denumerable infinity of eigenvalues $A$, which can be labelled by integers $l, m(|m|<l)$. Here and in the following we suppress the labels $s, l, m$ on $A, R, \lambda, S$, etc. where convenient. If a new independent variable $r^{*}$ is defined ${ }^{1}$ by

$$
\frac{d r^{*}}{d r}=\frac{r^{2}}{\Delta}
$$

then the region outside the horizon $\left(r_{+}<r<\infty\right)$ is mapped into the infinite line $\left(-\infty<r^{*}<\infty\right)$. Solutions of the Teukolsky equation which are regular on the horizon will be proportional to the solution with asymptotic behaviour

$$
R \rightarrow \Delta^{-s} e^{-i k r^{*}}, r^{*} \rightarrow-\infty,
$$

where $^{1} k=\left(2 M / r_{+}\right)\left(\omega-m \omega_{+}\right)$, and $\omega_{+}$is the angular velocity of the horizon, $\omega_{+}=a / 2 M r_{+}$. At infinity this solution will behave like (in the notation of Ref. [3])

$$
R \rightarrow Z_{\text {in }} e^{-i \omega r^{*}} / r+Z_{\text {out }} e^{i \omega r^{*}} / r^{(2 s+1)}, r^{*} \rightarrow+\infty .
$$

Following the discussion of Press and Teukolsky [2], a solution which corresponds to an instability in the Kerr geometry must lie in the upper half- $\omega$ plane in order that it have an amplitude which is growing in time. Further, it must be regular on the horizon and finite at infinity. Such solutions happen only for those values of $\omega$ in the upper half-plane where $Z_{\text {in }}$ vanishes. Press and Teukolsky show that such solution cannot exist by means of the following argument:

(1) Since the Schwarzschild geometry is stable [3], there can be no zeros of $Z_{\text {in }}$ in the upper half $\omega$ plane when $a=0$.

(2) As $a$ is increased, a zero can migrate into the upper half $\omega$ plane only by crossing the real axis.

(3) They then search the real axis numerically for zeros of $Z_{\text {in }}$ as $a$ is varied from 0 to $M$ and find none.

1 This variable $r^{*}$ originally used by J. Bardeen, W. Press and S. Teukolsky [Ap. J. 178, 347 (1972)], differs from that used in Ref. [2] but slightly simplifies some of the later discussion. Corresponding to this differing definition the boundary condition in Eq. (1.4) takes a slightly different form. 
The second step in the chain of argument is a crucial one. To justify it, it would be sufficient to show:

(i) That there are no zeros in the upper half-plane for some arbitrarily small but finite range of $a$ near zero.

(ii) That $Z_{\text {in }}$ was separately analytic in $\omega$ in the upper half plane and in $a$ in an arbitrarily small region surrounding the real $a$ axis between 0 and $M$.

(iii) That no zeros of $Z_{\text {in }}$ can move in from infinity as $a$ is increased from zero.

Then, since $Z_{\mathrm{in}}(\omega, a)$ is an analytic function of the variables $\omega$ and $a$ separately, by Hartogs' theorem it must be an analytic function of them jointly. Suppose that $\omega(a)$ is the trajectory of a zero of $Z_{\text {in }}$ as $a$ is varied along the real axis. For an analytic function of two complex variables, these trajectories, considered as curves in the three dimensional space $(\omega, a)$, can have no finite endpoints (Ref. [4], Chapter IV). Thus, as $a$ is varied, zeros of $Z_{\text {in }}$ cannot appear spontaneously in the $\omega$ plane. Taking account of (iii) above, this means that any zero of $Z_{\text {in }}$ in the upper-half plane could only have arrived there by crossing the real axis as $a$ is increased from zero.

By way of contrast, if there were branch points in the upper-half $\omega$ plane, there would always be the possibility that zeros could emerge through the associated cuts as $a$ is increased from 0 and wind up as unstable solutions in the upper-half $\omega$-plane without ever having crossed the real axis. The investigation of the analytic properties of the solutions to the Teukolsky equation thus becomes an important question in the analysis of the stability of the Kerr geometry. Of the above steps which are sufficient to show that all zeros cross the real axis, Press and Teukolsky [2] have already demonstrated (i) and (iii). In this paper we will investigate the analytic properties of $Z_{\text {in }}(\omega)$ necessary for step (ii). We will use the familiar techniques from the theory of the analytic properties of the scattering matrix in potential scattering. (For a small sample of this literature see Refs. $[5,6]$.) The general problem is substantially the same as in the theory of the one dimensional Schrödinger equation, as is shown in Section II. The only essential difference is that the separation constant, $A(a \omega)$, depends on $\omega$, and the analytic properties of $A$ as a function of $\omega$ are determined by the angular equation. These analytic properties are then reflected in the analytic properties of $Z_{\text {in }}$. Generalizing an argument given by Meixner and Shäfke [9] we show in Section III that in general $A$ will possess branch points where the eigenvalues of the angular equation become degenerate. These branch points of $A(\omega)$ will appear as branch points of $Z_{\text {in }}(\omega)$. The resulting branch cuts will not, however, vitiate the argument of Press and Teukolsky because, as we shall show in Section IV, the eigenvalues $A(\omega)$ reached by passing through 
these cuts for a particular mode are identical to those of the first sheet of a different mode. Aside from the cuts arising from $A(\omega)$, the only other cuts of $Z_{\text {in }}(\omega)$ will be shown to be a pair of branch cuts parallel to the imaginary axis. The fact that instabilities must appear in perturbations having both positive and negative spin weight will rule out any zeros emerging from these cuts. The conclusion is that the analytic properties derived here, taken together with the justificatory steps (i) and (iii) given above, and the numerical results of Press and Teukolsky are sufficient to demonstrate the stability of the Kerr geometry against perturbations in the modes calculated.

\section{Analytic Properties of the Radial Equation}

In this section we will derive the analytic properties of the coefficient $Z_{\text {in }}(\omega)$ defined in terms of the solution to the radial equation by Eq. (1.5). The analytic properties will be derived following standard potential scattering methods which are reviewed in (a) below. In this section the variables $\omega$ and $A$ will be treated as independent. Later (Section III) the explicit dependence of $A$ on $\omega$ will be considered.

(a) General Methods.

Writing $x$ instead of $r^{*}$ and introducing

$$
\psi(x)=\Delta^{s / 2} r R(r)
$$

the radial Teukolsky equation takes the general form

$$
\frac{d^{2} \psi}{d x^{2}}+F(x) \psi=0
$$

There are two linearly independent solutions $A_{ \pm}$of this equation which have the asymptotic behavior as $x \rightarrow+\infty$,

$$
A_{ \pm}(x) \rightarrow \Delta^{\mp s / 2} e^{ \pm i \omega x}, \quad x \rightarrow+\infty .
$$

There are also two linearly independent solutions $B_{ \pm}$which have the asymptotic behavior as $x \rightarrow-\infty$,

$$
B_{ \pm}(x) \rightarrow \Delta^{ \pm s / 2} e^{ \pm i k x}, \quad x \rightarrow-\infty .
$$

The coefficients $Z_{\text {in }}$ and $Z_{\text {out }}$ are then defined by the relation which expresses $B_{-}$in terms of the two linearly independent solutions $A_{ \pm}$.

$$
B_{-}(x)=\left[Z_{\text {in }} A_{-}(x)+Z_{\text {out }} A_{+}(x)\right] / r_{+} .
$$


To express the interesting coefficient $Z_{\text {in }}$ in terms of these solutions we define the Wronskian of two solutions $\psi, \chi$ of Eq. (2.2), by

$$
W[\psi, \chi]=\psi \frac{d \chi}{d x}-\chi \frac{d \psi}{d x} .
$$

It follows from the differential equation that the Wronskian of any two solutions is independent of $x$. In particular

$$
W\left[A_{-}, A_{+}\right]=2 i \omega .
$$

Using this, we can write

$$
Z_{\text {in }}=\frac{r_{+}}{2 i \omega} W\left[B_{-}, A_{+}\right]
$$

This is the relation which will be used to establish the analytic properties of $Z_{\text {in }}$ by establishing the analytic properties of $A_{+}$and $B_{-}$.

To investigate the solutions $A_{+}$or $B_{-}$we break $F$ in Eq. (2.2) up into a sum of two pieces $F_{0}$ and $F_{1}$ such that the equation

$$
\frac{d^{2} \psi_{0}}{d x^{2}}+F_{0} \psi_{0}=0
$$

can be solved explicitly. Let $\psi_{0}^{(1)}$ and $\psi_{0}^{(2)}$ be any two linearly independent solutions of this equation. Its Green's function can then be written

$$
G\left(x, x^{\prime}\right)=N\left[\psi_{0}^{(1)}(x) \psi_{0}^{(2)}\left(x^{\prime}\right)-\psi_{0}^{(2)}(x) \psi_{0}^{(1)}\left(x^{\prime}\right)\right]
$$

where the normalization constant $N$ is given by

$$
N^{-1}=W\left[\psi_{0}^{(2)}, \psi_{0}^{(1)}\right] \text {. }
$$

To construct a solution of the full equation [Eq. (2.2)] with a given asymptotic behavior at $x=+\infty$, one now proceeds as follows: Pick a solution of Eq. (2.9) which has the desired asymptotic behavior. (For this to be possible, $F_{1}$ must approach zero at $x=+\infty$ more rapidly than $F_{0}$.) The solution of the full equation which has the same asymptotic _ shavior can be found by solving the integral equation

$$
\psi(x)=\psi_{0}(x)+\int_{x}^{\infty} G\left(x, x^{\prime}\right) F_{1}\left(x^{\prime}\right) \psi\left(x^{\prime}\right) d x^{\prime} .
$$

Solutions defined by boundary conditions at $x=-\infty$ can be constructed in a similar way with the upper limit replaced by $-\infty$. Equations of the type of Eq. (2.12) can be solved by iteration. The resulting series is generally convergent since the equation is essentially of Volterra type.

To investigate the analytic properties of $A_{+}$and $B_{-}$, we will construct integral equations for them of the above type and solve these equations 
by iteration. The result will be a series each term of which will be analytic in certain domains of the variables $\omega, a$, and $A$. The series will be shown to converge uniformly in these domains, yielding domains of analyticity for $A_{+}$and $B_{-}$. Where necessary, these domains can be extended using the technique of contour rotation (Appendix A). The analytic properties of $Z_{\text {in }}$ will then follow directly from Eq. (2.8).

As far as the analyticity in the variable $a$ is concerned we will be interested only in an arbitrarily small domain surrounding the real axis between 0 and $M-\varepsilon$ where $\varepsilon$ is arbitrarily small. For brevity we will refer to this domain as $D_{a}$.

(b) The Solution $B_{-}(x)$.

The explicit expression for $F$ in Eq. (2.2) is

$$
\begin{aligned}
F(x)=\frac{2 \Delta^{2}}{r^{6}} & -\frac{s^{2}(r-M)^{2}}{r^{4}}-\frac{2 \Delta(r-M)}{r^{5}}-\frac{s \Delta}{r^{4}} \\
& +\frac{1}{r^{4}}\left[K^{2}-2 i s(r-M) K+\Delta(4 i r \omega s-\lambda)\right]
\end{aligned}
$$

where $r$ is a function of $x$ defined implicitly for $a<M$ by

$$
x(r)=r+M \log \left(\frac{\Delta}{a^{2}}\right)+\frac{\left(2 M^{2}-a^{2}\right)}{2\left(M^{2}-a^{2}\right)^{\frac{1}{2}}} \log \left(\frac{r r_{-}-a^{2}}{r r_{+}-a^{2}}\right) .
$$

To apply the above procedure to the solution $B_{-}$, we note that near $r=r_{+}$

$$
F=\Omega^{2}+0\left[\left(r-r_{+}\right)\right]
$$

where

$$
\Omega=\frac{2 M}{r_{+}}\left(\omega-\frac{a m}{2 M r_{+}}\right)-\frac{i s\left(r_{+}-M\right)}{r_{+}^{2}} .
$$

For $F_{0}$ we take the constant $\Omega^{2}$ and it then follows that the solutions to the free equation [Eq. (2.9)] are of the form $\exp ( \pm i \Omega x)$. The integral equation defining $B_{-}(x)$ is

$$
B_{-}(x)=e^{-i \Omega x}-\int_{-\infty}^{x} \frac{\sin \left[\Omega\left(x-x^{\prime}\right)\right]}{\Omega} F_{1}\left(x^{\prime}\right) B_{-}\left(x^{\prime}\right) d x^{\prime} .
$$

Iterating this equation we find the series

$$
B_{-}(x)=e^{-i \Omega x}+\sum_{n=1}^{\infty} B_{-}^{(n)}(x)
$$


where, with $B_{-}^{(0)}(x)=e^{-i \Omega x}$,

$$
\begin{aligned}
B_{-}^{(n)}(x)=- & \int_{-\infty}^{x} d x^{\prime} \frac{\sin \left[\Omega\left(x-x^{\prime}\right)\right]}{\Omega} F_{1}\left(x^{\prime}\right) B_{-}^{(n-1)}\left(x^{\prime}\right) \\
B_{-}^{(n)}(x)= & (-1)^{n} \int_{-\infty}^{x} d x_{1} \int_{-\infty}^{x_{1}} d x_{2} \ldots \int_{-\infty}^{x_{n}-1} d x_{n} \\
& \cdot \frac{\sin \left[\Omega\left(x-x_{1}\right)\right]}{\Omega} \frac{\sin \left[\Omega\left(x_{n-1}-x_{n}\right)\right]}{\Omega} \\
& x F_{1}\left(x_{1}\right) \ldots F_{1}\left(x_{n}\right) e^{-i \Omega x_{n}} .
\end{aligned}
$$

The various elements of this expression can be bounded as follows:

$$
\begin{gathered}
\left|e^{-i \Omega x}\right|=e^{\Omega_{I} x} \\
\left|\frac{\sin \Omega y}{\Omega}\right| \leqq g(y) e^{\left|\Omega_{I}\right| y}, \quad y>0
\end{gathered}
$$

where a subscript $I$ denotes the imaginary part of that quantity, and

$$
g(y)=\frac{2 y}{1+|\Omega| y} .
$$

Since $g(y)$ is monotonically increasing for $y>0$ we also have

$$
g\left(x_{k-1}-x_{k}\right) \leqq g\left(x-x_{k}\right) .
$$

Let $b$ be any positive number and consider values of $\omega$ such that

$$
\Omega_{I}>-b / 2 \text {. }
$$

Further let us restrict the range of $x$ under consideration to $-\infty \leqq x \leqq x_{0}$, where $x_{0}$ is arbitrary but positive. It follows that

$$
e^{x_{n}\left(\Omega_{I}-\left|\Omega_{I}\right|\right)} \leqq e^{b\left(x_{0}-x_{n}\right)}
$$

Making use of the above bounds and symmetrizing the integrand, one can bound $B_{-}^{(n)}(x)$ by

where

$$
\left|B_{-}^{(n)}(x)\right| \leqq \frac{e^{\left|\Omega_{I}\right| x}}{n !}[\alpha(x)]^{n}
$$

$$
\alpha(x)=\int_{-\infty}^{x} d x^{\prime} g\left(x-x^{\prime}\right)\left|F_{1}\left(x^{\prime}\right)\right| e^{b\left(x_{0}-x^{\prime}\right)} .
$$

At large negative $x$,

$$
F_{1}(x) \rightarrow \text { const } \cdot e^{c x}
$$


where

$$
c=\left(r_{+}-r_{-}\right) / r_{+}^{2}
$$

The largest value of $b$ for which the integral defining $\alpha(x)$ will exist is therefore $c$. Equivalently, the integral defining $\alpha(x)$ exists provided

$$
\Omega_{I}>-\sqrt{M^{2}-a^{2}} / r_{+}^{2},
$$

or

$$
\omega_{I}>(s-1) \frac{\sqrt{M^{2}-a^{2}}}{2 M r_{+}} .
$$

The bound (2.24) and Eq. (2.25) can be used to show that $B_{-}^{(n)}(x)$ is an entire function of $A$ and an analytic function of $\omega$ and $a$ for $\omega$ restricted by Eq. (2.27) and $a \in D_{a}$. This follows from a theorem of analysis which states that a function $G(z)$ represented by

$$
G(z)=\int_{-\infty}^{x} g(z, r) d r
$$

will be analytic in $z$ if the integrand is analytic in $z$, continuous in $r$ and the integral converges uniformly at its lower limit. The integral converges uniformly for $\omega, a$, and $A$ in bounded sub-domains of the domains specified above, by Eqs. (2.24) and (2.25). Noting that $B_{-}^{(0)}\left(x^{\prime}\right)$ and $\sin \Omega\left(x-x^{\prime}\right) F_{1}\left(x^{\prime}\right) / \Omega$ are continuous in $x^{\prime}$ and analytic in $\omega, a$, and $A$, one can now prove inductively that $B_{-}^{(n)}(x)$ is analytic in the same domain.

The bound in Eq. (2.24) shows that the series in Eq. (2.18) will converge uniformly in any bounded subdomains of the domains described by $A$ arbitrary, $\omega$ restricted by Eq. $(2.27 \mathrm{~b})$, and $a \in D_{a}$. We conclude that $B_{-}(x)$ is an analytic function in this domain.

The above demonstration is not quite sufficient for our purposes since, if $s>1$, the region defined by Eq. $(2.27 \mathrm{~b})$ does not include the whole upper half $\omega$ plane. The domain of analyticity can be extended by standard contour rotation techniques used in potential scattering. Since the proof is so similar to the potential scattering case we will only quote the results here and relegate a concise derivation to Appendix $\mathrm{A}$. The result is that the function $B_{-}(x)$ is an entire function of $A$, analytic in the $\omega$ plane except for a cut beginning at

$$
\omega=m \omega_{+}+i(s-1) \frac{\left(r_{+}-M\right)}{2 M r_{+}}
$$

and running in the negative imaginary direction parallel to the imaginary axis, and is analytic in $a$ in $D_{a}$. 
(c) The Solution $A_{+}(x)$.

The solution $A_{+}(x)$ can be handled in essentially the same manner as $B_{-}(x)$. At large positive $x$, one has

$$
F(x)=\omega^{2}+\frac{2 i \omega s}{x}+0\left(\frac{\log (x)}{x^{2}}\right) .
$$

For $F_{0}(x)$, we will take $\omega^{2}+2 i \omega s / x$. Two linearly independent solutions of the free equation can be taken to be

$$
\begin{aligned}
& \psi_{0}^{(-)}=W_{s, \frac{1}{2}}(2 i \omega x), \\
& \psi_{0}^{(+)}=W_{-s, \frac{1}{2}}(-2 i \omega x),
\end{aligned}
$$

where $W_{k, m}(z)$ is the Whittaker function [8], which satisfies the differential equation

$$
\left(\frac{d^{2}}{d z^{2}}-\frac{1}{4}+\frac{k}{z}+\frac{1 / 4-m^{2}}{z^{2}}\right) W_{k, m}(z)=0,
$$

and has the asymptotic behavior

$$
W_{k, m}(z)=z^{k} e^{-z / 2}\left[1+0\left(\frac{1}{z}\right)\right] .
$$

Using these two independent solutions, the Green's function may be constructed according to Eq. (2.10):

$$
\begin{aligned}
G\left(x, x^{\prime}\right)= & \frac{(-1)^{s}}{2 i \omega}\left[W_{-s, \frac{1}{2}}(-2 i \omega x) W_{s, \frac{1}{2}}\left(2 i \omega x^{\prime}\right)\right. \\
& \left.-W_{-s, \frac{1}{2}}\left(-2 i \omega x^{\prime}\right) W_{s, \frac{1}{2}}(2 i \omega x)\right] .
\end{aligned}
$$

The integral equation for $A_{+}(x)$ is then

with

$$
A_{+}(x)=A_{+}^{(0)}(x)+\int_{x}^{\infty} G\left(x, x^{\prime}\right) F_{1}\left(x^{\prime}\right) A_{+}\left(x^{\prime}\right) d x^{\prime},
$$

$$
A_{+}^{(0)}(x)=(-2 i \omega)^{s} W_{-s, \frac{1}{2}}(-2 i \omega x),
$$

chosen so that

$$
A_{+}^{(0)}(x) \rightarrow x^{-s} e^{i \omega x}, \quad x \rightarrow+\infty .
$$

It is apparent from the asymptotic behavior of the solutions to the free equation that the integral in the above equation exists for real values of $\omega$ only in the case $s \geqq 0$. This reflects the fact that for real $\omega$ and $s<0$, the asymptotic behavior in Eq. (2.35) does not uniquely define a solution because an arbitrary amount of the linearly independent solution with large $x$ behavior $x^{s} \exp (-i \omega x)$ could be added in without affecting this 
asymptotic behavior. If $\omega$ has a small positive imaginary part, however, the integral will converge and define a unique solution which will have a well defined limit as $\omega$ approaches the real axis. In the following for $s<0$, we will assume $\omega_{I}>0$.

We now proceed as with $B_{-}(x)$. Iterating the equation one finds

$$
A_{+}(x)=A_{+}^{(0)}(x)+\sum_{n=1}^{\infty} A_{+}^{(n)}(x),
$$

with

$$
\begin{aligned}
A_{+}^{(n)}(x) & =\int_{x}^{\infty} d x_{1} \int_{x_{1}}^{\infty} d x_{2} \ldots \int_{x_{n-1}}^{\infty} d x_{n} G\left(x, x_{1}\right) G\left(x_{1}, x_{2}\right) \\
& \ldots G\left(x_{n-1}, x_{n}\right) F_{1}\left(x_{1}\right) \ldots F_{1}\left(x_{n}\right) A_{+}^{(0)}\left(x_{n}\right) .
\end{aligned}
$$

Let us consider first the case $s \geqq 0$. From the analytic properties of the Whittaker functions derived in Appendix B, it follows for $x>0$ and $x^{\prime}>0$ that $A_{+}^{(0)}(x)$ and $G\left(x, x^{\prime}\right)$ are analytic in $\omega$ in the upper half plane $\left(\omega_{I} \geqq 0\right)$. Utilizing Eqs. (2.41), (2.42) the integrals in Eq. (2.38) can be shown to be uniformly convergent in the variables $\omega, A$, and $a$, in any bounded subdomain of the region specified by $\omega_{I} \geqq 0, A$ arbitrary, and $a \in D_{a}$. It then follows that $A_{+}^{(n)}(x)$ is analytic in the same domain. There remains the demonstration that the series in Eq. (2.37) converges uniformly.

From the bounds on the Whittaker functions which are derived in Appendix B, one has in the case $s \geqq 0, x^{\prime}>x$

$$
\begin{gathered}
\left|A_{+}^{(0)}(x)\right| \leqq e^{-\omega_{I} x}|2 \omega x|^{-s} \\
\left|G\left(x, x^{\prime}\right)\right| \leqq \frac{s !}{|\omega|} e^{\omega_{I}\left(x^{\prime}-x\right)}\left(\frac{\left|2 \omega x^{\prime}\right|+1}{|2 \omega x|}\right)^{s} .
\end{gathered}
$$

Following the outline of the proof for $B_{-}(x)$, one can then show

$$
\left|A_{+}^{(n)}(x)\right| \leqq \frac{1}{n !} \frac{e^{-\omega_{I} x}}{|2 \omega x|^{s}}[\alpha(x)]^{n}
$$

where

$$
\alpha(x)=\frac{s !}{|\omega|} \int_{x}^{\infty} d x^{\prime}\left(\frac{\left|2 \omega x^{\prime}\right|+1}{\left|2 \omega x^{\prime}\right|}\right)^{s}\left|F_{1}\left(x^{\prime}\right)\right| .
$$

From Eq. (2.30), $F_{1}\left(x^{\prime}\right)$ is $O\left[\log (x) / x^{2}\right]$, so the integral in Eq. (2.42) always converges. The series in Eq. (2.37) then converges uniformly in a closed, bounded domain of $(\omega, A, a)$ for non-zero $\omega$ such that $\omega_{I} \geqq 0$, $A$ arbitrary and $a \in D_{a}$. We then have the result that $A_{+}(x)$ must be analytic in this domain. 
The case $s<0$ is slightly different. There we find from Appendix B that, while $A_{+}^{(0)}(x)$ is an entire function of $\omega, G\left(x, x^{\prime}\right)$, for $x>0$ and $x^{\prime}>0$, has a branch point at $\omega=0$. This branch point will therefore occur in the $A_{+}^{(n)}$ defined through Eq. (2.38). As previously discussed, when $s<0$, we consider only the region $\omega_{I} \geqq \varepsilon>0$, excluding the origin, in particular. Provided $\omega_{R} \neq 0$, one has the following bounds on $A_{+}^{(0)}$ and $G\left(x, x^{\prime}\right)$ :

$$
\begin{aligned}
&\left|A_{+}^{(0)}(x)\right| \leqq|s| ! e^{-\omega_{I} x}(|2 \omega x|+1)^{|s|} \\
&\left|G\left(x, x^{\prime}\right)\right| \leqq \frac{|s| !}{2|\omega|}\left|\frac{\omega}{\omega_{R}}\right|^{|s|+1}\left\{e^{\omega_{I}\left(x^{\prime}-x\right)}\left(\frac{|2 \omega x|+1}{\left|2 \omega x^{\prime}\right|}\right)^{|s|}\right. \\
&\left.+e^{\omega_{I}\left(x-x^{\prime}\right)}\left(\frac{\left|2 \omega x^{\prime}\right|+1}{|2 \omega x|}\right)^{|s|}\right\} .
\end{aligned}
$$

It is not difficult to show, using Eq. (2.44), that when

$$
\begin{gathered}
x^{\prime} \geqq x \geqq|s| / \varepsilon, \\
\left|G\left(x, x^{\prime}\right)\right| \leqq \frac{|s| !}{|\omega|}\left|\frac{\omega}{\omega_{R}}\right|^{|s|+1} e^{\omega_{I}\left(x^{\prime}-x\right)}\left(\frac{|2 \omega x|+1}{\left|2 \omega x^{\prime}\right|}\right)^{-s} .
\end{gathered}
$$

Following the methods used for $B_{-}(x)$, one finds

$$
\left|A_{+}^{(n)}(x)\right| \leqq \frac{|s| ! e^{-\omega_{I} x}}{n !}(|2 \omega x|+1)^{|s|}[\alpha(x)]^{n}
$$

where

$$
\alpha(x) \equiv \frac{|s| !}{|\omega|}\left|\frac{\omega}{\omega_{R}}\right|^{|s|+1} \int_{x}^{\infty} d x^{\prime}\left|F_{1}\left(x^{\prime}\right)\right|\left(\frac{\left|2 \omega x^{\prime}\right|+1}{\left|2 \omega x^{\prime}\right|}\right)^{|s|} .
$$

Since $F_{1}\left(x^{\prime}\right)$ is $O\left[\log (x) / x^{2}\right]$, the integral in Eq. (2.47) converges. For $x$ sufficiently large, the series defining $A_{+}(x)$ therefore converges uniformly in any closed, bounded domain of $(\omega, A, a)$ satisfying $\omega_{I}>0, \omega_{R} \neq 0$ and $a \in D_{a} ; x$ is "sufficiently large" for the given set of $\omega$ values if it exceeds the finite positive number $|s| / \min \left(\omega_{I}\right) . A_{+}(x)$ is then analytic in this domain of $\omega, A$ and $a$. By rotating the contour, as for $B_{-}$(see Appendix A), $A$ can be analytically continued to all $\omega$, excepting a branch point at $\omega=0$. The branch cut is conveniently taken along the negative imaginary axis. $A_{+}$for $s<0$ is therefore analytic for all non-zero $\omega$ with $\omega_{I} \geqq 0$.

\section{The Analytic Properties of the Separation Constants}

In the previous section it has been shown that $Z_{\text {in }}(\omega, A)$ is an analytic function of $\omega$ and $A$ considered as independent variables. The functional dependence of $A$ on $\omega$ is determined by the angular equation and will be 
discussed in this section by generalizing slightly the methods given in Meixner and Shäfke [7] for spheroidal harmonics.

Introducing the independent variable $z=\cos \theta$, a new separation constant $A^{*}=A+s$, and $\varrho=a \omega$, the angular equation [Eq. (1.2)] reads

$$
\frac{d}{d z}\left[\left(1-z^{2}\right) \frac{d S}{d z}\right]+\left[\varrho^{2} z^{2}-2 \varrho s z-\frac{(m+s z)^{2}}{1-z^{2}}+A^{*}\right] S=0 \text {. }
$$

This is a second order differential equation with regular singular points at $z= \pm 1$. Near $z=-1$, there is a regular solution with the behavior

$$
S \sim(z+1)^{|m-s| / 2} .
$$

This behavior is independent of $A^{*}$. This regular solution at $z=-1$ can be expanded in a power series about that point. As the coefficients in the equation are polynomials in $\varrho$ and $A^{*}$, it follows from the elementary theory of power series solutions to differential equations (see Ref. [7], Section 1.3) that the series converges uniformly in any bounded domain in both $\varrho$ and $A^{*}$, for $z$ in the range $-1 \leqq z<1$. Since this domain can be chosen as large as one pleases, the solution $S$ is an entire function of $\varrho$ and $A^{*}$ considered as separate complex variables.

The eigenvalues $A^{*}(\varrho)$ are those values of $A^{*}$ which make this solution $S$ finite at $z=+1$. The eigenvalue condition can, however, be put in a more usable form. For given $s, \varrho, m$ and $A^{*}$, suppose there exist solutions $S(s, z)$ and $S(-s, z)$ regular at $z=-1$ and satisfying either of the symmetries

$$
S(-S,-z)= \pm S(s, z) \text {. }
$$

It follows that both solutions are also regular at $z=+1$, which is to say, $A^{*}$ is an eigenvalue. Conversely if $A^{*}$ is an eigenvalue, $S(S, z)$ will be finite at $z= \pm 1$. Noting the invariance of Eq. (3.1) under the simultaneous transformation $s \rightarrow-s, z \rightarrow-z$, it follows that the eigensolutions will either have one of the symmetries of Eq. (3.3) or can be chosen to have one of them in the case the eigenvalue is degenerate. The eigenvalue condition is thus equivalent to the existence of a pair of solutions satisfying one of the symmetry conditions of Eq. (3.3) and regular at $z=-1$.

Consider power series solutions $S$ which are regular at $z=-1$. If we define $\Delta_{ \pm}$and $D_{ \pm}$by

$$
\begin{gathered}
\Delta_{ \pm}\left(A^{*}, \varrho\right)=\left.\left.S\left(A^{*}, \varrho, s, z\right)\right|_{z=0} \mp S\left(A^{*}, \varrho,-s, z\right)\right|_{z=0}, \\
D_{ \pm}\left(A^{*}, \varrho\right)=\left.\frac{d S}{d z}\left(A^{*}, \varrho, s, z\right)\right|_{z=0} \pm\left.\frac{d S}{d z}\left(A^{*}, \varrho,-s, z\right)\right|_{z=0},
\end{gathered}
$$


the conditions $\Delta_{+}=D_{+}=0\left(\Delta_{-}=D_{-}=0\right)$ imply that Eq. (3.3) holds with a plus (minus) sign, and consequently that $A^{*}$ is an eigenvalue. Dropping the requirements $D_{ \pm}=0$ further enlarges the set of solutions $A^{*}$. There are thus a pair of functions $\Delta_{ \pm}\left(A^{*}, \omega\right)$ separately analytic in $A^{*}$ and $\varrho$, and vanishing for a set of values $A^{*}(\varrho)$, among which are all the eigenvalues. From the implicit function theorem [4] it then follows that the solutions $A^{*}(\varrho)$ are analytic functions of $\varrho$ except possibly where $\partial \Delta_{ \pm} / \partial A^{*}$ vanishes. Such points will be branch points or singularities of the functions $A^{*}(\varrho)$.

The analytic properties of $A^{*}(\varrho)$ can now be defined. Choose a particular mode characterized by integers $l$ and $m$. The first sheet of the function $A^{*}(\varrho)$ is defined to be that for which $A^{*}(0)=l(l+1)-s^{2}$ and the first few terms in a power series about $\varrho=0$ are those given by Press and Teukolsky [2]. The function $A^{*}(\varrho)$ is thus analytic on the first sheet except for the branch points and singularities described above. The branch points can occur wherever there are a multiplicity of solutions of the equations $\Delta_{ \pm}=0$ having the same value of $A^{*}$ for given $\omega$, i.e., they occur whenever the eigenvalues are degenerate. Since all the eigenvalues are labeled by some value of the integer $l$, passing through the branch cut one must reach a sheet identical with the first sheet of some different integer $l^{2}$. The second and higher order sheets thus contain no new information not found on the collection of first sheets, considering all values of $l$.

\section{Conclusions}

We have established that $B_{-}(x)$ and $A_{+}(x)$ are entire functions of $A$ and analytic in $a$ in the domain $D_{a}$. The function $B_{-}(x)$ is an analytic function of $\omega$ except for a cut running parallel to the imaginary axis starting from a point given in Eq. (2.27b). The function $A_{+}(x)$ is analytic in $\omega$ in the entire upper half $\omega$-plane. It then follows from Eq. (2.8) that $Z_{\text {in }}$ will be an entire function of $A$, analytic in $a$ in $D_{a}$ and analytic in $\omega$ in the upper half plane with the exception of the cut in $B_{-}$. If we now restrict $A$ to the function of a $\omega$ whose properties were derived in the previous section, we find $Z_{\text {in }}$ is analytic in $a$ for $a \in D_{a}$ and analytic in the upper half $\omega$-plane except for the singularities of $A(\varrho)$ and the branch points of $A(\varrho)$ and $B_{-}(x)$.

In the introduction, it was argued that the analyticity of $Z_{\text {in }}$ in $D_{a}$ and in the upper-half $\omega$ plane, together with several other properties would be sufficient to justify Press and Teukolsky's demonstration of stability. In regard to the analyticity in $\omega$, however, we have not completely

\footnotetext{
2 We are grateful to W. Press for discussions of this point.
} 
excluded singularities and branch points from the upper half plane. Our results, however, are still sufficient to complete Press and Teukolsky's argument.

The possible singularities of $A(\omega)$ which could appear as singularities of $Z_{\text {in }}$ will not affect the stability argument since that is concerned with the zeros of $Z_{\text {in }}$. Any cuts of $A(\omega)$ reflected in $Z_{\text {in }}$ only connect the first sheets of a given mode $(l, m)$ to the first sheet of a different mode $\left(l^{\prime}, m\right)$. Thus no zero of $Z_{\text {in }}$ could emerge from one of these cuts without first crossing the real axis for some mode, and the results of Press and Teukolsky make this implausible.

There remains the possibility that zeros could emerge from the cut of $B_{-}$without first crossing the real axis. The work of Wald [9], however, has shown us that instabilities in the Kerr geometry must occur both in the spin-weight +2 and spin-weight -2 parts of the Riemann tensor. There must be, therefore, a correspondence between the zeros of $Z_{\text {in }}(\omega)$ for $s=+2$ and those of $s=-2$. Since the cut in the upper-half plane present in the $s=+2$ function is absent in the $s=-2$, no zeros can possibly emerge from this cut without first having crossed the real axis as $a$ is increased from zero. Thus while we have not verified the simple analyticity properties conjectured by Press and Teukolsky, it appears that the analyticity present is sufficient to justify their argument.

Acknowledgements. Thanks are due to W. Press and R. Sugar for helpful discussions.

\section{Appendix A: Contour Rotation and an Extended Domain of Analyticity for $B_{-}(x)$}

In this Appendix, we will briefly illustrate the use of the method of contour rotation in extending the domain of analyticity of the function $B_{-}(x)$. We follow closely the standard potential scattering proof given in Ref. [5]. Consider Eq. (2.2) for values of $x$ along a rotated contour defined by

$$
x=\varrho e^{i \varphi}, \quad-\infty<\varrho<\infty, \quad|\varphi|<\pi / 2 .
$$

Making use of Eq. (2.15) the equation along this contour can be written

where

$$
\frac{d^{2} \psi}{d \varrho^{2}}+\left[\tilde{\Omega}^{2}+\tilde{F}_{1}(\varrho, \tilde{\Omega})\right] \psi=0
$$

and

$$
\tilde{\Omega}=e^{i \varphi} \Omega
$$

$$
\tilde{F}_{1}=e^{2 i \varphi} F_{1}\left(\varrho e^{i \varphi}, \tilde{\Omega} e^{-i \varphi}\right) .
$$

We can now define a function $\tilde{B}_{-}(\varrho, \tilde{\Omega})$ analogous to $B_{-}(x, \Omega)$ by an equation similar to Eq. (2.17). 
The mapping from $x$ to $r$ needed to define $\tilde{F}_{1}$ is not determined unambiguously by $x(r)$, Eq. (2.14), for complex values of $x$ and $r$. This occurs because, in general, many $r$ values may correspond to a single $x$. For $\varphi=0, r(x)$ is uniquely defined. A unique $r(x)$ may be singled out when $\varphi \neq 0$ by requiring that for $\varrho$ fixed, $r\left(\varrho e^{i \varphi}\right)$ shall vary continuously with $\varphi$. One can then show that $x=0$ on every rotated contour maps into the same positive real value of $r$. The additive constant in $x(r)$ was so chosen that $r=0$ maps into a set of images on the imaginary $x$ axis (depending on the phases of $r-r_{+}$and $r-r_{-}$). It follows that along any rotated contour, $r(x)$ never vanishes and, consequently, $\tilde{F}_{1}$ never blows up. The asymptotic behavior of $\tilde{F}_{1}$ along a rotated contour can be determined if one notes that when $\varrho \rightarrow+\infty, r(x) \rightarrow \infty$ and when $\varrho \rightarrow-\infty$, $r(x)$ spirals into $r=r_{+}$. One easily finds that $\tilde{F}_{1}(\varrho, \tilde{\Omega})$ decreases exponentially at large negative values of $\varrho$ provided $|\varphi|<\pi / 2$ :

$$
F_{1}(\varrho) \rightarrow \text { const } \cdot e^{c \varrho \cos (\varphi)},
$$

where $c$ is given by Eq. (2.26b). It then follows that $\tilde{B}_{-}(\varrho, \tilde{\Omega})$ is analytic for $\tilde{\Omega}_{I}>-\frac{1}{2} c \cos \varphi$.

A second solution, $\tilde{B}_{+}$, of Eq. (A.2), analogous to $B_{+}$, can be defined, with the asymptotic behavior $\tilde{B}_{+}(\varrho, \tilde{\Omega}) \rightarrow \exp [i \tilde{\Omega} \varrho]$ as $\varrho \rightarrow-\infty$. Because $F_{1}(x, \Omega)$ is an analytic function of $x$ in the domain given by Eq. (A.1), the solution $B_{-}(x, \Omega)$ can be analytically continued to these values of $x$, and in particular to the rotated contour. Because $B_{-}$satisfies Eq. (A.2), it must be expressible as a linear combination of $\tilde{B}_{+}$and $\tilde{B}_{-}$:

$$
B_{-}(x, \Omega)=\alpha(\varphi) \tilde{B}_{+}(\varrho, \tilde{\Omega})+\beta(\varphi) \tilde{B}_{-}(\varrho, \tilde{\Omega}) .
$$

Now, there is a region in the $\Omega$ plane where both $\Omega_{I}>0$ and $\tilde{\Omega}_{I}>0$. If $\tilde{\Omega}$ lies in this region $\tilde{B}_{-}(\varrho, \tilde{\Omega})$ will fall off exponentially as $\varrho$ goes to $-\infty$. Therefore, noting that $\Omega x=\tilde{\Omega} \varrho$,

$$
\lim _{\varrho \rightarrow-\infty}\left[\exp (-i \Omega x) B_{-}(x, \Omega)\right]=\alpha(\varphi) .
$$

Now by Montel's theorem (see Ref. [5], p. 11), since the function inside square brackets in Eq. (A.5) is analytic and bounded in $x$ inside the wedge $|\varphi| \leqq \pi / 2$, it must have the same limit for every value of $\varphi$ inside the wedge. In other words,

$$
\alpha(\varphi)=\alpha(0)=0 .
$$

Using this fact, multiplying Eq. (A.4) by $\exp (i \Omega x)$, and repeating the argument, it follows that $\beta(\varphi)=1$. Thus,

$$
B_{-}(x, \Omega)=\tilde{B}_{-}(\varrho, \tilde{\Omega}) .
$$

Equation (A.7) provides an extension of the domain of analyticity of $B_{-}$ to the union of the domains of analyticity of $B_{-}$and $\tilde{B}_{-}$. It is not difficult 
to verify that if $\varphi$ takes all values in the interval $|\varphi|<\pi / 2$, this domain is the whole of the $\Omega$ plane with the exception of the imaginary $\Omega$ axis between

$$
-\infty<\Omega_{I}<-\frac{\sqrt{M^{2}-a^{2}}}{r_{+}^{2}} .
$$

As we see here, the problem of finding the analytic properties of $B_{-}$ as a function of $\Omega$ is essentially the same as finding those of the Jost function in potential scattering with an exponentially decreasing potential. From these latter studies, a cut along the negative imaginary axis is known to exist. The whole $\Omega$ plane, with the exception of Eq. (A.8), is therefore probably the maximal domain of analyticity.

\section{Appendix B: Bounds on the Whittaker Functions}

Whittaker and Watson [8] give the following integral representations for the function $W_{k, \frac{1}{2}}(z)$. When $k$ is an integer $\leqq 0$

$$
W_{k, \frac{1}{2}}(z)=\frac{e^{-z / 2} z^{k}}{(-k) !} \int_{0}^{\infty} t^{-k}(1+t / z)^{k} e^{-t} d t
$$

and when $k$ is an integer $>0$,

$$
W_{k, \frac{1}{2}}(z)=-(k-1) ! e^{-z / 2} z^{k} \frac{1}{2 \pi i} \oint_{C}(-t)^{-k}\left(1+\frac{t}{z}\right)^{k} e^{-t} d t
$$

where the contour $C$ runs in from $+\infty$ below the real axis, around $t=0$ and out to $t=+\infty$ above the real axis. Using these two integral representations, we can derive bounds on the Whittaker functions. When $k \leqq 0$, the two cases $z_{R} \geqq 0$ and $z_{R}<0$ must be considered separately. For $z_{R} \geqq 0$, we can bound the integral by using

$$
\left|1+\frac{t}{z}\right| \geqq 1
$$

and the integral representation for the gamma function. One finds

$$
\left|W_{k, \frac{1}{2}}(z)\right| \leqq\left|e^{-z / 2} z^{k}\right|
$$

This bound holds independently of any restriction on $z_{R}$ when $k=0$. If $z_{R}<0$ but $z_{I} \neq 0$, a sufficiently stringent bound is obtained by first rotating the contour in Eq. (B.1) through an angle $\varphi$ given by

$$
\tan \varphi=\frac{\left|z_{R}\right|}{z_{I}}, \quad|\varphi|<\pi / 2 .
$$


The value of the integral is preserved because the rotating contour does not pass through the singularity of the integrand at $t=-z$. Bounding the resulting integrand as in Eq. (B.3), one thus obtains for $k \leqq 0$ and $z_{R}<0$,

$$
\left|W_{k, \frac{1}{2}}(z)\right| \leqq\left|e^{-z / 2} z^{k}\right|\left|z / z_{I}\right|^{|k|+1} .
$$

In the case that $k$ is a positive integer, the integral in Eq. (B.2) can be evaluated to give a finite series

$$
W_{k, \frac{1}{2}}(z)=(k-1) ! e^{-z / 2}(-z)^{k} \sum_{i=0}^{k-1}\left(\begin{array}{l}
k \\
i
\end{array}\right) z^{-i} \frac{(-1)^{k-1-i}}{(k-1-i) !} .
$$

Just using $(k-1-i) ! \geqq 1$ and $\left(\begin{array}{l}k \\ i\end{array}\right) \leqq k\left(\begin{array}{c}k-1 \\ i\end{array}\right)$, this can be bounded by

$$
\left|W_{k, \frac{1}{2}}(z)\right| \leqq k !\left|e^{-z / 2}\right|(|z|+1)^{k} .
$$

Comparison with Eq. (B.4) shows that this inequality also holds for $k=0$. These bounds are used in Section II(c).

Minimal analytic properties of the Whittaker functions follow from Eq. (B.6) and the integral representation in Eq. (B.1). When $k$ is an integer greater than zero, we have from Eq. (B.6) that $W_{k, \frac{1}{2}}(z)$ is an entire function of its argument $z$. Since the integral in Eq. (B.1) exists provided $z$ is not real and negative, it follows that $W_{k, \frac{1}{2}}(z)$ is analytic in $z$ except for the real negative axis when $k<0$ and is an entire function of $z$ when $k=0$.

\title{
References
}

1. Teukolsky, S. A.: Phys. Rev. Lett. 29, 1114 (1972); Ap. J. 185, 635 (1973)

2. Press, W., Teukolsky, S.: Ap. J. 185, 649 (1973)

3. Vishveshwara, C.V.: Phys. Rev. D, 1, 2870 (1970)

4. Bochner, S., Martin, W. T.: Several complex variables. Princeton: Princeton University Press 1948

5. de Alfaro,V., Regge, T.: Potential scattering. Amsterdam: North-Holland Pub. Co. 1965

6. Newton, R.: J. Math. Phys. 1, 319 (1960)

7. Meixner, J., Shäfke,F.W.: Mathieusche Funktionen und Sphäroidfunktionen. BerlinGöttingen-Heidelberg: Springer 1954

8. Whittaker, E., Watson, G.: A course of modern analysis, 4 th ed., Chapter XVI. Cambridge: 1927

9. Wald, R.: J. Math. Phys. 14, 1453 (1973)

Communicated by J. Ehlers

\author{
J. B. Hartle \\ D. C. Wilkins \\ Department of Physics \\ University of California \\ Santa Barbara, California 93106, USA
}


